\title{
Transforming growth factor- $\beta 1$ regulates human renal proximal tubular epithelial cell susceptibility to natural killer cells via modulation of the NKG2D ligands
}

\author{
HYUNKEUN SONG ${ }^{1}$, YEONYE KIM ${ }^{1}$, GABIN PARK ${ }^{2}$, YEONG-SEOK KIM ${ }^{2}$, SEONGHAN KIM ${ }^{2}$, \\ HYUN-KYUNG LEE ${ }^{3}$, WOO YEONG CHUNG ${ }^{4}$, SEOK JU PARK ${ }^{3}$, \\ SANG-YOUB HAN ${ }^{5}$, DAEHO $\mathrm{CHO}^{6}$ and DAEYOUNG HUR ${ }^{2}$
}

\begin{abstract}
Departments of ${ }^{1}$ Microbiology and Immunology, Laboratory for Medical Oncology and ${ }^{2}$ Anatomy, Inje University College of Medicine; Departments of ${ }^{3}$ Internal Medicine, ${ }^{4}$ Pediatrics, Inje University Busan Paik Hospital, Busan 614-735; ${ }^{5}$ Department of Internal Medicine, Inje University Ilsan-Paik Hospital, Goyang, Gyeonggi 411-706;

${ }^{6}$ Department of Life Science, Sookmyung Women's University, Yongsan-ku, Seoul 140-742, Republic of Korea
\end{abstract}

Received January 16, 2015; Accepted August 13, 2015

DOI: $10.3892 /$ ijmm.2015.2317

\begin{abstract}
Transforming growth factor- $\beta$ (TGF- $\beta$ ) has a significant role in the response to injury and tissue repair, and it has been detected in various cell types. However, the mechanism by which it regulates the response to ischemia-reperfusion injury (IRI) and manipulates natural killer (NK) cells is not well understood. In the present study, TGF- $\beta$ modulated NK cell function, thereby promoting recovery from renal IRI. Human renal proximal tubular epithelial cells $(\mathrm{HK}-2)$ treated with TGF- $\beta$ exhibited increased surface and intracellular expression of the NK group 2 member D (NKG2D) ligand MICA. This increased surface expression of MICA inhibited NK cell cytotoxicity to the HK-2 cells. In addition, an enzyme-linked immunosorbent assay revealed that TGF- $\beta$ treatment evidently increased the amount of soluble MICA released into the culture supernatant from HK-2 cells. Taken together, these findings suggest that TGF- $\beta$-induced release of soluble MICA leads to downregulation of NKG2D, thereby preventing NK cell-mediated cytotoxicity toward renal proximal tubular epithelial cells in renal IRI, which in turn improves the survival of these cells.
\end{abstract}

Correspondence to: Professor Daeyoung Hur, Department of Anatomy, Inje University College of Medicine, 633-165 Kaekum-2dong, Jin-gu, Busan 614-735, Republic of Korea

E-mail:dyhur@inje.ac.kr

Abbreviations: TGF- $\beta$, transforming growth factor- $\beta$; NK, natural killer; IRI, ischemia-reperfusion injury; TECs, tubular epithelial cells; NKG2D, NK group 2 member D; PBLs, peripheral blood lymphocytes; FACS, fluorescence-activated cell sorting; FITC, fluorescein isothiocyanate; ATM, ataxia telangiectasia mutated; ATR, ATM- and Rad3-related

Key words: tubular epithelial cells, transforming growth factor- $\beta$, natural killer cell, ischemia-reperfusion injury, NK group 2 member D

\section{Introduction}

Ischemia-reperfusion injury (IRI), in particular renal IRI, is an unavoidable event and a critical clinical problem in organ transplantation. Renal IRI is a major cause of kidney graft dysfunction eventually leading to graft loss $(1,2)$. IRI is also involved in graft malfunction following transplantation in cardiac and aortic surgery $(3,4)$. The physiological response of tubular epithelial cells (TECs) has a pivotal role in kidney IRI etiology (5-7), which involves a cascade of events, including oxidative stress and inflammation that in turn trigger wound healing by restoration of blood flow to the ischemic tissue $(6,8)$. Following ischemia, oxidative stress leads to the production of tumor necrosis factor- $\alpha$ and interferon- $\gamma$, cytokines associated with TEC injury $(9,10)$. Following exposure to these cytokines, TECs trigger kidney malfunction $(5,6)$.

Accumulating evidence suggests that kidney IRI is a complex event involving innate and adaptive immune cells, including cluster of differentiation $4^{+}\left(\mathrm{CD}^{+}\right) \mathrm{T}$ cells, $B$ cells, resident dendritic cells, natural killer (NK) cells and neutrophils (11-13), although kidney IRI can develop in $\mathrm{T}$ and B cell-deficient Rag1 ${ }^{-/}$mice $(14,15)$. The present study focused on the role of innate immune cells, specifically NK cells, in renal IRI. Normally, NK cells recognize and lyse virus-infected and tumor cells; however recently, NK cells have been shown to be involved in renal IRI through direct killing of TECs $(16,17)$. In mice, induced expression of the NK group 2 member D (NKG2D) ligand Rae-1 on TECs has been observed during kidney IRI (18). From these observations, NK cells appear to be major participants in kidney IRI.

NK cell activity is triggered by its recognition of a target cell through integration of signals from inhibitory and activating receptors $(19,20)$. For example, activation of NK cells is dependent on NK cell expression of NKG2D, an activating receptor, and tumor cell expression of NKG2D ligands, together with the presence of a danger signal, such as cellular stress and/or viral infection (21). In humans, the NKG2D ligands major histocompatibility complex class I-related chain 
molecules A and B (MICA/B) and UL16-binding proteins (ULBPs), are upregulated in response to various signals, including cellular and genotoxic stress, but are not expressed under normal conditions. The interaction of MICA/B and NKG2D on NK cells triggers the cytotoxic function of NK cells. Thus, NKG2D ligands are considered useful therapeutic targets for the elimination of tumor and virus-infected cells by NK cells $(22,23)$. Certain studies have reported that tumor cells can produce transforming growth factor- $\beta$ (TGF- $\beta$ ) leading to a decrease in surface NKG2D expression on NK and $\mathrm{CD}^{+} \mathrm{T}$ cells $(24,25)$.

TGF- $\beta$ is a well-characterized cytokine that can induce cell apoptosis, proliferation and differentiation (26). Certain studies of renal IRI have found that TGF- $\beta$ expression is strongly induced in renal tubules, as well as in biopsies of kidney grafts following ischemic injury $(27,28)$. In addition, several studies have reported that TGF- $\beta$ detected in TECs was a signal indicating recovery in post-ischemic kidneys $(29,30)$; however, the functional implications of upregulated TGF- $\beta$ expression in TECs during renal IRI are not clear.

Notably, certain previous studies have reported that TECs produce TGF- $\beta$ following renal IRI, suggesting that TGF- $\beta$ may inhibit NK cell activation via downregulation of the NKG2D receptor. By contrast, other studies have shown that NK cells kill TECs through upregulation of NKG2D ligands during renal IRI $(18,31)$. On the basis of these observations, we hypothesized that TGF- $\beta$ regulates NKG2D ligand expression in renal IRI. In the present study, whether the expression of NKG2D ligands on human TECs (HK-2) is regulated by TGF- $\beta$ was investigated. The functional consequences of TGF- $\beta$-induced NKG2D ligand expression on TECs during the interaction between TECs and NK cells were also evaluated. The results of the present study examining TGF- $\beta$ modulation of the NK cell-TEC interaction in renal IRI may lead to novel insights and useful therapeutic approaches to treat renal inflammatory injury, including renal transplantation.

\section{Materials and methods}

Cell culture and reagents. The human renal proximal TEC line (HK-2) was purchased from American Type Culture Collection (Manassas, VA, USA) and cultured in Dulbecco's modified Eagle medium (Invitrogen, Carlsbad, CA, USA) with non-essential amino acids, $0.05 \mathrm{mg} / \mathrm{ml}$ bovine pituitary extract, $50 \mathrm{ng} / \mathrm{ml}$ human recombinant epidermal growth factor, $100 \mathrm{U} / \mathrm{ml}$ penicillin, $100 \mu \mathrm{g} / \mathrm{ml}$ streptomycin and $10 \%$ fetal bovine serum (FBS) under a $5 \% \mathrm{CO}_{2}$ atmosphere at $37^{\circ} \mathrm{C}$. NK92-MI cells were cultured in $\alpha$-modification of Eagle's Minimum Essential medium supplemented with $2 \mathrm{mM}$ L-glutamine, $0.2 \mathrm{mM}$ inositol, $20 \mathrm{mM}$ folic acid, 12.5\% FBS and $12.5 \%$ horse serum. Anti-NKG2D antibodies (\#FAB139A) and anti-NKG2D ligand antibodies (\#MAB13001,\#MAB1380, \#MAB1298, \#MAB1517) were purchased from R\&D Systems (Minneapolis, MN, USA).

Isolation and culture of human peripheral blood lymphocytes. Peripheral blood lymphocytes (PBLs) from buffy coats of healthy donors were prepared by Ficoll-Hypaque density gradient centrifugation (GE Healthcare, Little Chalfont, UK). The study was approved by the Institutional Bioethics Review
Board at the Medical College of Inje University (Busan, Korea), and all the donors provided informed written consent prior to their participation in the study. Cells were stimulated with human interleukin (IL)-2 (500 U/ml; a quantity commonly used to stimulate primary NK cells) for cytokine production (32).

Flow cytometry analysis. Surface antigen fluorescence-activated cell sorting (FACS) analysis was performed to detect NKG2D ligands on HK-2 cells. Cells were washed twice with ice-cold phosphate-buffered saline (PBS), and incubated with mouse anti-MICA antibody (\#MAB13001) or anti-human ULBP monoclonal antibodies [anti-ULBP1 (\#MAB1380), anti-ULBP2 (\#MAB1298) and anti-ULBP3 (\#MAB1517); R\&D Systems $\}$ for 30 min on ice. After 2 washes, cells were incubated with an appropriate fluorescein isothiocyanate (FITC)-conjugated secondary antibody diluted in PBS for $30 \mathrm{~min}$ on ice and analyzed using a FACSCalibur flow cytometer.

Intracellular FACS analysis was performed to detect intracellular NKG2D ligand protein levels in HK-2 cells. Cells were washed twice with ice-cold PBS containing $0.05 \%$ bovine serum albumin (BSA) and $0.02 \%$ sodium azide (PBS-BSA), and were fixed in $2 \%$ paraformaldehyde in PBS for $15 \mathrm{~min}$ on ice. The cells were subsequently washed once in cold PBS-BSA, and resuspended in PBS containing 0.1\% saponin and $0.05 \%$ sodium azide (permeabilization buffer) for $15 \mathrm{~min}$, followed by incubation with anti-MICA and anti-ULBPs antibodies for $30 \mathrm{~min}$ on ice. Following 2 washes, cells were incubated with an appropriate FITC-conjugated secondary antibody in permeabilization buffer for $30 \mathrm{~min}$ on ice. Samples were analyzed using a FACSCalibur flow cytometer (BD Biosciences, Franklin. Lakes, NJ, USA).

Reverse transcription-polymerase chain reaction (RT-PCR). RNA was isolated from HK-2 cells using RNeasy (Qiagen, Valencia, CA, USA), according to the manufacturer's instructions, and reverse transcribed into cDNA. The following primers were used for PCR amplification: TGF- $\beta$ sense, 5'-GCC CTG GAC ACC AAC TAT TGC-3' and antisense, 5'-GCT GCA CTT GCA GGA GCG CAC-3'; and MICA sense, 5'-TGC TTC TGG CTG GCA TCT TC-3' and antisense, 5'-TAG TTC CTG CAG GCA GTC TG-3'. Cycling conditions for MICA and TGF- $\beta$ were $1 \mathrm{~min}$ at $94^{\circ} \mathrm{C}, 1 \mathrm{~min}$ at $60^{\circ} \mathrm{C}$ and $1 \mathrm{~min}$ at $72^{\circ} \mathrm{C}$ for 32 cycles. $\beta$-actin was amplified as a control using a sense, 5'-GTG GGG CGC CCC AGG CAC CA-3' and antisense primer, 5'-CTC CTT AAT GTC ACG CAC GAT TTC-3'; in the RT-PCR experiments. Cycling conditions for $\beta$-actin were $1 \mathrm{~min}$ at $94^{\circ} \mathrm{C}, 1 \mathrm{~min}$ at $55^{\circ} \mathrm{C}$ and $1 \mathrm{~min}$ at $72^{\circ} \mathrm{C}$ for 25 cycles. The PCR products were analyzed by ethidium bromide-stained $1.2 \%$ agarose gel electrophoresis

Small interfering RNA (siRNA) transfection. TGF- $\beta$ expression was blocked by siRNA. Cell transfection was performed using Lipofectamine 2000 (Invitrogen) for $48 \mathrm{~h}$. The siRNA sequences were as follows: TGF- $\beta$ siRNA sense, 5'-CAG AGU ACA CAC AGC AUA U-dTdT-3' and antisense, 5'-AUA UGC UGU GUG UAC UCU G-dTdT-3'; and non-related control siRNA (GL3-luciferase) for TGF- $\beta$ siRNA transfection sense, 5'-AGG GAU ACA UAC GUG CAC G-dTdT-3' and antisense, 5'-CGU GCA CGU AUG UAU CCC U-dTdT-3'. RT-PCR 
A Surface expression

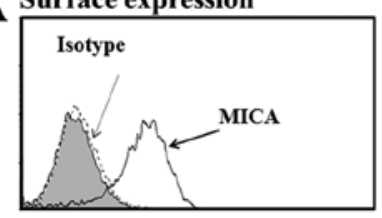

Intracellular expression

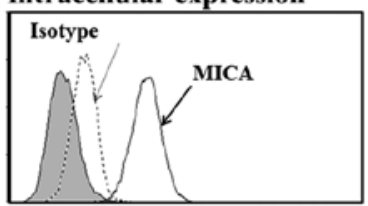

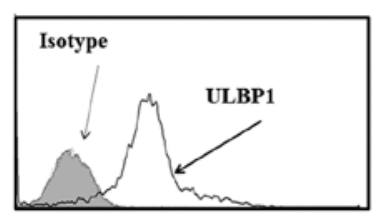

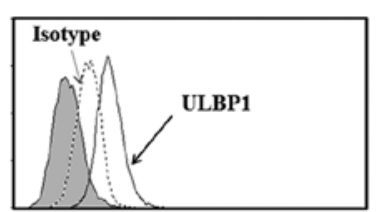

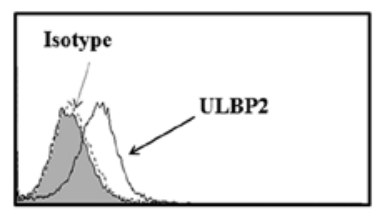
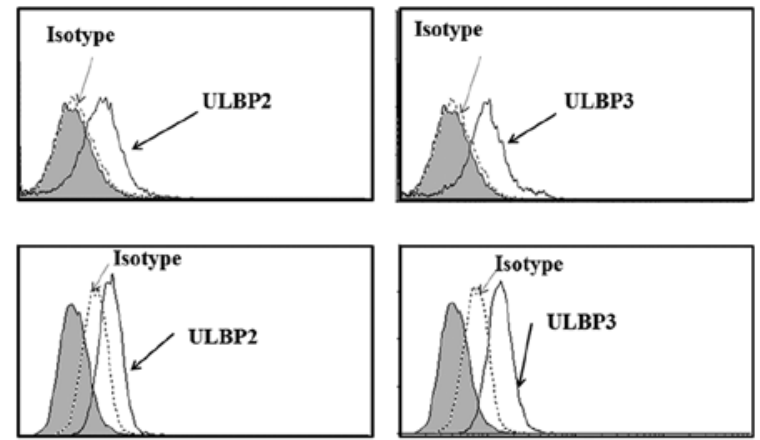

B Surface expression
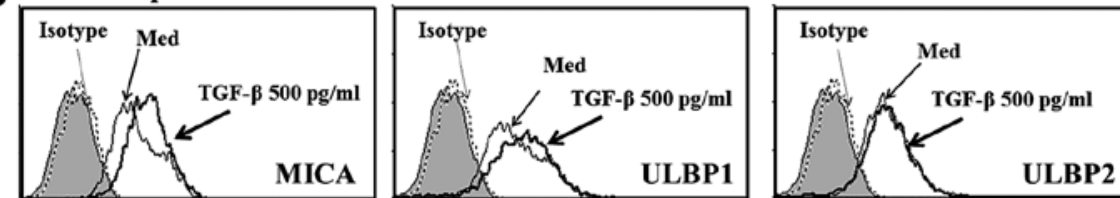

Intracellular expression
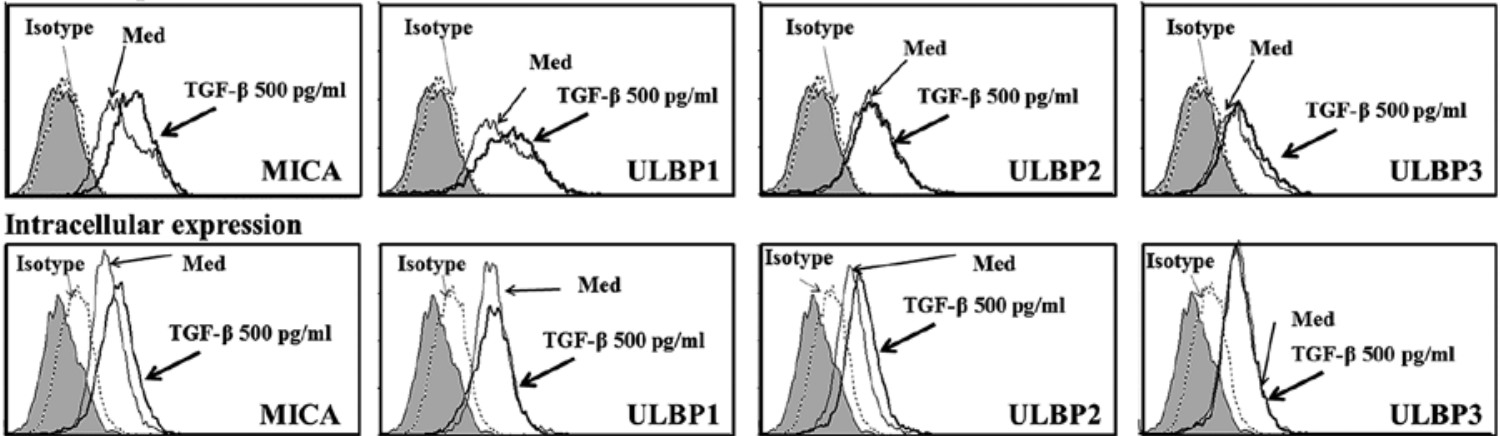

Figure 1. Expression of NKG2D ligands by renal proximal tubular epithelial cell. (A) Surface and intracellular staining of NKG2D ligand proteins. Human renal proximal TECs (HK-2) were stained with control normal mouse immunoglobulin G (iso) or anti-MICA, and anti-ULBP1, 2 or 3 antibodies, and analyzed by flow cytometry. In the histograms, the gray peak represents the unstained control. (B) TGF- $\beta$-induced expression of NKG2D ligands. Renal proximal TECs (HK-2) were incubated in the presence of TGF- $\beta(500 \mathrm{pg} / \mathrm{ml})$ for $48 \mathrm{~h}$, and surface and intracellular expression of NKG2D ligands were analyzed by flow cytometry. Results are representative of three independent experiments. NKG2D, NK group 2 member D; TECs, tubular epithelial cells; MICA, major histocompatibility complex class I-related chain molecules A; ULBP, UL16-binding proteins; TGF, transforming growth factor.

was performed to determine the level of silencing following transfection.

NK cell cytotoxicity assays. NK cell cytotoxic activity was determined by calcein-AM assays, as previously described (33). Briefly, target cells were washed twice with PBS and incubated with $5 \mathrm{mM}$ calcein-AM (Molecular Probes, Eugene, OR, USA) in serum-free RPMI-1640 medium for $10 \mathrm{~min}$ at $37^{\circ} \mathrm{C}$. Labeled target cells were distributed into U-bottom microtiter plates at a concentration of $2 \times 10^{4}$ cells/well. Effector cells (PBL or the NK92MI NK cell line) were added at various effector to target ratios in quadruplicate. Target cells (HK-2 cells) in complete RPMI-1640 medium alone were used to determine spontaneous calcein-AM retention. Maximal lysis was determined by solubilizing 3 wells of the target cells in lysis buffer (0.1\% Triton X-100). After incubation for $5 \mathrm{~h}$, the assays were analyzed using a fluorescence reader. The percentage of specific cytotoxicity was calculated as follows: $\%$ specific release $=[$ (retention of experimental well - retention of spontaneous well)/(retention of maximal lysis well - retention of spontaneous well)] x100.

Enzyme-linked immunosorbent assay (ELISA). The concentrations of TGF- $\beta$ and soluble MICA in culture supernatants were determined using human TGF- $\beta$ and MICA ELISA kits (R\&D Systems) following the manufacturer's instructions. Briefly, cell culture supernatant was added to microplate wells coated with either anti-TGF- $\beta$ or anti-MICA antibody.
After incubation with the cultured supernatant for $2 \mathrm{~h}$, the wells were washed 4 times with washing buffer. Horseradish peroxidase-conjugated detection antibodies were added for $2 \mathrm{~h}$, followed by the addition of the substrate solution for $30 \mathrm{~min}$. Samples were read at $450 \mathrm{~nm}$ with an ELISA reader (Molecular Devices, Sunnyvale, CA, USA).

Statistical analysis. Statistical analysis was performed using GraphPad Prism (GraphPad Software, La Jolla, CA, USA). Student's t-test was used for assessing statistical significance for comparisons between two groups. Intergroup differences were assessed by one-way analysis of variance. $\mathrm{P}<0.05$ was considered to indicate a statistically significant difference.

\section{Results}

TGF- $\beta$ enhances MICA expression on renal proximal TECs. To evaluate NKG2D ligand expression in human renal proximal tubular epithelial (HK-2) cells, surface and intracellular expression of MICA and ULBP was analyzed. HK-2 cells expressed MICA, ULBP2 and ULBP3, but not ULBP1, on the cell surface. However, all these NKG2D ligands were detected intracellularly (Fig. 1A).

Several studies have reported TGF- $\beta$ expression in renal proximal TECs (30), and in general, TGF- $\beta$ has been shown to inhibit NK cell function via downregulation of NKG2D $(24,25,34)$. To investigate the association between expression of NKG2D ligands and TGF- $\beta$ in renal IRI, 
A Surface expression

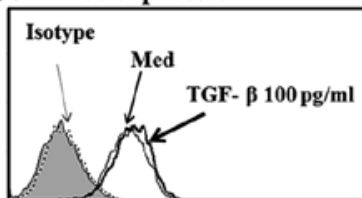

Intracellular expression
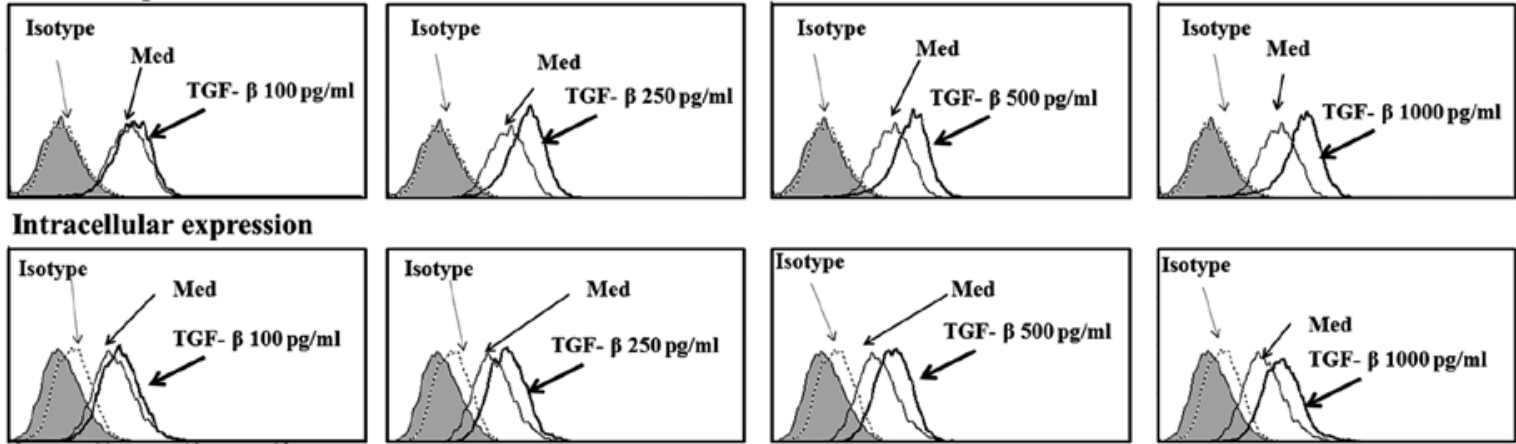

B Surface expression
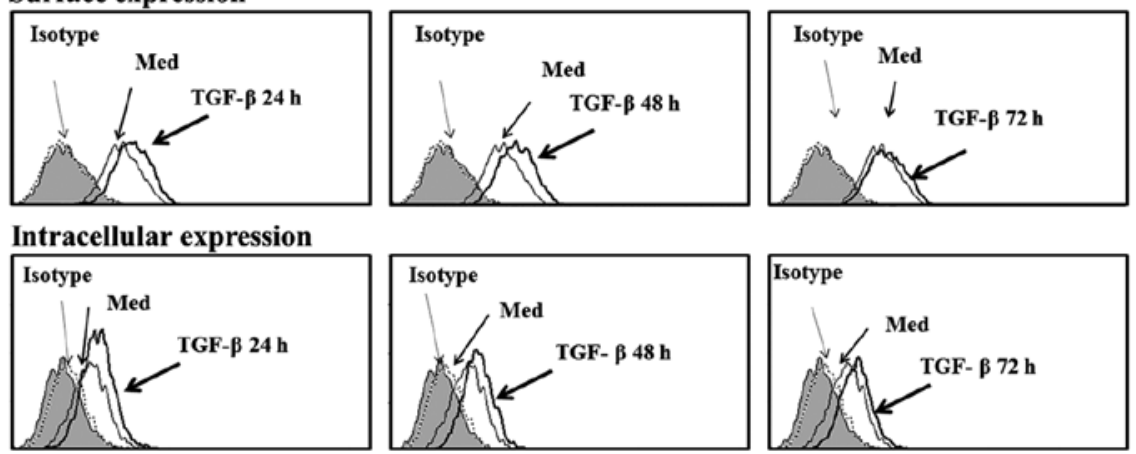

Figure 2. TGF- $\beta$ induces surface and intracellular expression of MICA on renal proximal tubular epithelial cells in a dose- and time-dependent manner (A) Surface and intracellular staining of MICA proteins following treatment of HK-2 cells with various concentrations of TGF- $\beta$ for 48 h. Human renal proximal TECs cells were analyzed for surface and intracellular concentrations of MICA proteins, as described in the Material and methods section. The gray shaded area represents the unstained control cells, and the dotted line represents the secondary antibody control. MICA basal expression is represented by the thin line. The bold line indicates TGF- $\beta$-enhanced MICA expression in HK- 2 cells. These data represent 1 of 3 independent experiments. (B) Surface and intracellular staining of MICA proteins. HK-2 cells were incubated with TGF- $\beta$ ( $500 \mathrm{pg} / \mathrm{ml})$. At various time-points, the cells were washed with cold phosphate-buffered saline, stained with control normal mouse immunoglobulin G (iso) or anti-MICA antibody, and analyzed by flow cytometry. The gray shaded area represent the unstained control cells, and the dotted line represents the secondary antibody control. MICA basal expression is represented by the thin line. The bold line indicates TGF- $\beta$-enhanced MICA expression in HK- 2 cells. Results are representative of three independent experiments. TGF, transforming growth factor; MICA, major histocompatibility complex class I-related chain molecules A; TECs, tubular epithelial cells.

NKG2D ligand expression was measured using FACS analysis following treatment with exogenous TGF- $\beta$. Treatment with exogenous TGF- $\beta$ induced the intracellular expression of MICA and ULBP2 in HK-2 cells; however, it did not induce the intracellular expression of other NKG2D ligands (Fig. 1B). Notably, treatment with TGF- $\beta$ induced MICA surface expression in HK-2 cells, but induced little or no surface expression of ULBP2.

To confirm these findings, the effects of different concentrations of TGF- $\beta$ on MICA expression in HK-2 cells were evaluated. TGF- $\beta$ treatment significantly induced surface and intracellular expressions of MICA in a dose-dependent manner (Fig. 2A). Time-course experiments revealed that $48 \mathrm{~h}$ of incubation in the presence of TGF- $\beta$ was optimal for the maximum induction of intracellular and surface expression of MICA in HK-2 cells (Fig. 2B). These results show that MICA expression increases in HK-2 cells following treatment with TGF- $\beta$ in a dose- and time-dependent manner. Taken together, these data suggest that TGF- $\beta$ is a regulator of MICA expression in human TECs.

TGF- $\beta$ regulates expression of MICA in renal proximal TECs via the ataxia telangiectasia mutated (ATM)/ATM- and Rad3-related (ATM/ATR) pathway. Several studies in various cell types have revealed that NKG2D ligand expression is primarily controlled by the ATM and ATR protein kinase pathway $(35,36)$. Kirshner et al (37) reported that TGF- $\beta$ is involved in the ATM/ATR kinase pathway in response to genotoxic stress. To determine which pathway mediates the upregulation of MICA in HK-2 cells, an ATM/ATR kinase inhibitor was tested for its ability to block MICA expression following treatment with TGF- $\beta$. The ATM/ATR kinase inhibitor caffeine significantly blocked the expression of MICA induced by TGF- $\beta$ (Fig. 3A). Additionally, ATM/ATR kinase activity and ATR kinase phosphorylation were markedly increased in HK-2 cells following treatment with TGF- $\beta$ (Fig. 3B). These results suggest that the ATM/ATR pathway has a critical role in controlling the expression of MICA following TGF- $\beta$ treatment.

Functional consequences of TGF- $\beta$-induced soluble and membrane-bound MICA expression in HK-2 cells. To examine whether membrane-bound MICA expression on HK-2 cells influences cytotoxic function in primary NK cells, the cytotoxicity of PBL, isolated from healthy individuals, against HK-2 cells treated with or without TGF- $\beta$ was evaluated. IL-2-activated effector cells exhibited significantly higher cytotoxicity against HK- 2 cells treated with TGF- $\beta$ compared 

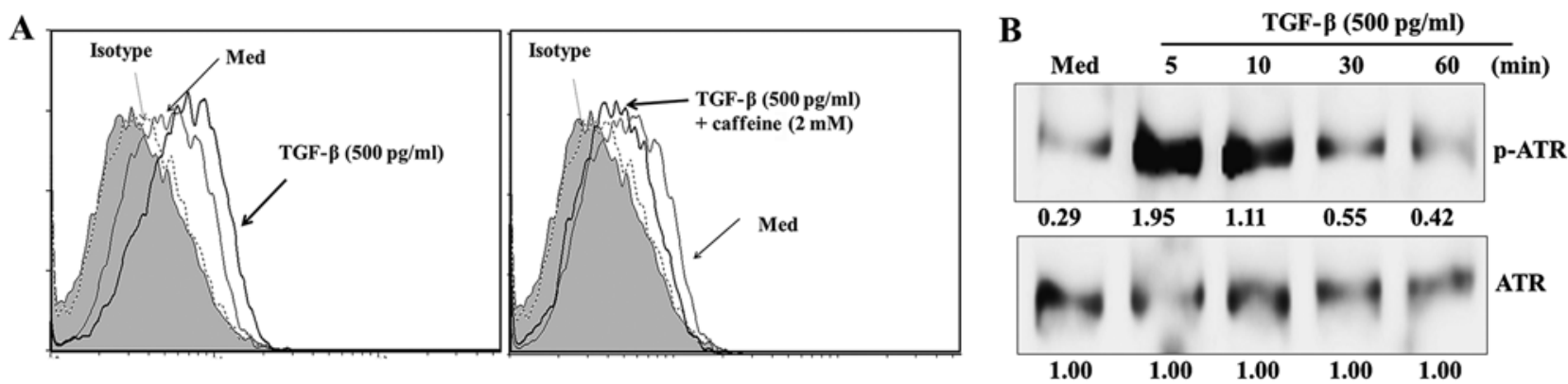

Figure 3. TGF- $\beta$ promotes surface expression of MICA on renal proximal tubular epithelial cells via the ATM/ATR pathway. (A) Surface staining of MICA proteins following treatment with an ATM/ATR kinase inhibitor and TGF- $\beta(500 \mathrm{pg} / \mathrm{ml})$ for $48 \mathrm{~h}$. Human renal proximal tubular epithelial cells were pretreated with or without $2 \mathrm{mM}$ of caffeine (an ATM/ATR kinase inhibitor) for $1 \mathrm{~h}$. Following washing, cells were incubated with or without TGF- $\beta$ (500 pg/ml) for $48 \mathrm{~h}$. MICA expression was subsequently analyzed by surface fluorescence-activated cell sorting staining with anti-human MICA antibody. (B) ATR kinase immunoblot from renal proximal tubular epithelial cells treated with TGF- $\beta(500 \mathrm{pg} / \mathrm{ml})$. Expression levels of phospho-ATR and ATR were evaluated by western blot analysis. Results are representative of three independent experiments. TGF, transforming growth factor; MICA, major histocompatibility complex class I-related chain molecules A; ATM/ATR, ataxia telangiectasia mutated (ATM)/ATM- and Rad3-related.

$\mathbf{A}$
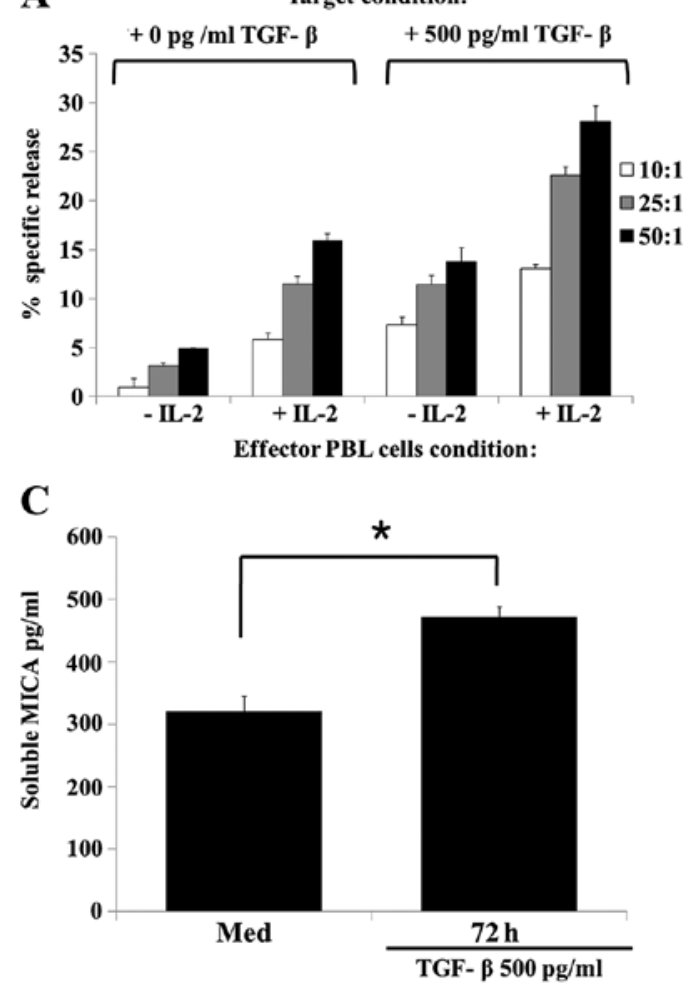

B

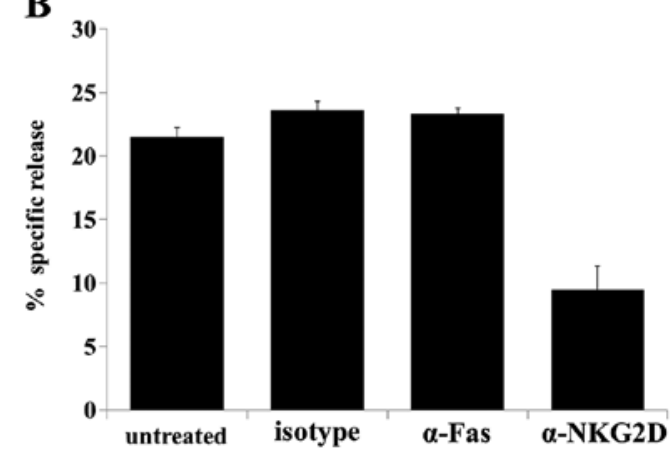

D

Target condition :

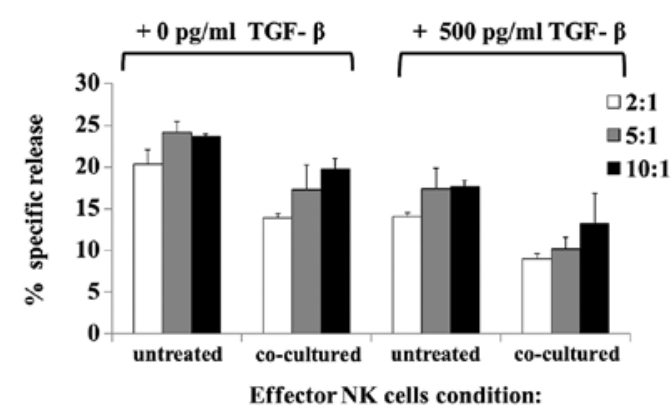

Figure 4. TGF- $\beta$ treatment increased HK-2 cells susceptibility to NK-mediated lysis via NKG2D mediated pathway. (A) PBL cytotoxicity induced by treatment of HK-2 cells with TGF- $\beta$ (500 pg/ml) for $48 \mathrm{~h}$. HK-2 cells were incubated with or without TGF- $\beta$ (500 pg/ml) for 48 h. Target cells were subsequently harvested, labeled with calcein-AM for $10 \mathrm{~min}$, washed with phosphate-buffered saline, and loaded at an effector to target cell ratio of 50:1 (black-filled bars), 25:1 (gray-filed bars), and 10:1 (open bars). After a 4-h incubation period, the specific lysis activity of the PBL was analyzed using a fluorescence reader. (B) Cytotoxicity of PBL against HK-2 [treated with TGF- $\beta$ (500 pg/ml)] was assessed after pre-incubating PBL (5x10 6 cells/well) with IL-2 (500 U/ml) for $24 \mathrm{~h}$. PBL cytotoxicity was induced by treatment of HK-2 cells with TGF- $\beta$ ( $500 \mathrm{pg} / \mathrm{ml})$ for 48 h. Target HK-2 cells were incubated with TGF- $\beta$ (500 pg/ml) for $48 \mathrm{~h}$. Target cells were harvested, labeled, washed and loaded at an effector to target cell ratio of 50:1, as described above. PBL and target cell mixtures were subsequently cultured with the indicated antibodies $(1 \mu \mathrm{g} / \mathrm{ml})$ for an additional $4 \mathrm{~h}$. (C) HK-2 cells were treated with or without TGF- $\beta$ (500 pg/ml) for $48 \mathrm{~h}$. The MICA secretion level in culture supernatants was analyzed by an enzyme-linked immunosorbent assay. (D) Cytotoxicity of NK92-MI cells against HK-2 [treated with or without TGF- $\beta(500 \mathrm{pg} / \mathrm{ml})$ ] was assessed after pre-incubating NK92MI cells (2x10 6 cells/well) with complete media (untreated) or with TGF- $\beta(500 \mathrm{pg} / \mathrm{ml})$ plus HK-2 cells $\left(2 \times 10^{6}\right.$ cells/well) for $48 \mathrm{~h}$. TGF- $\beta$ treated and untreated HK- 2 cells and NK cells were co-cultured using a Transwell system. The HK-2 cells treated or untreated with TGF- $\beta$ for $48 \mathrm{~h}$ were used as the target cells. Results are representative of three independent experiments. ${ }^{*} \mathrm{P}<0.05$ vs. control. TGF, transforming growth factor; NKG2D, NK group 2 member D; PBL, peripheral blood lymphocytes; IL, interleukin; MICA, major histocompatibility complex class I-related chain molecules A.

with the naïve HK-2 cells (Fig. 4A). Subsequently, whether the observed TGF- $\beta$-mediated increase in the susceptibility of HK-2 cells to NK cell cytotoxicity directly involves
NKG2D was examined. Neutralizing NKG2D with an anti-NKG2D antibody moderately reduced PBL cytotoxicity against TGF- $\beta$-treated HK-2 target cells, while treatment with 
A
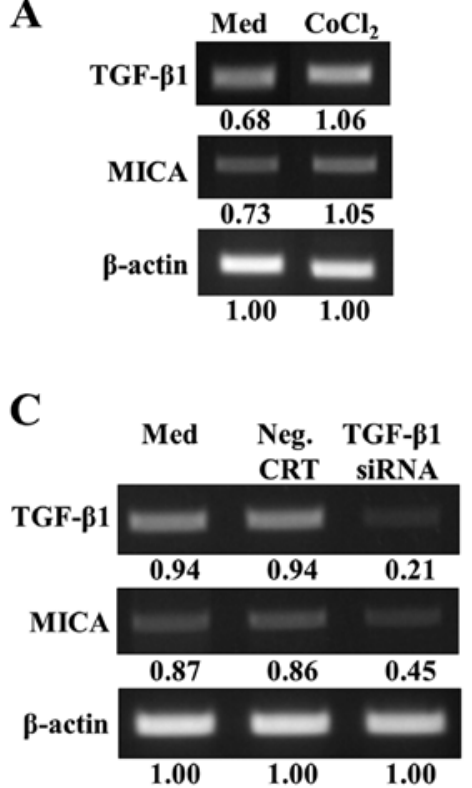

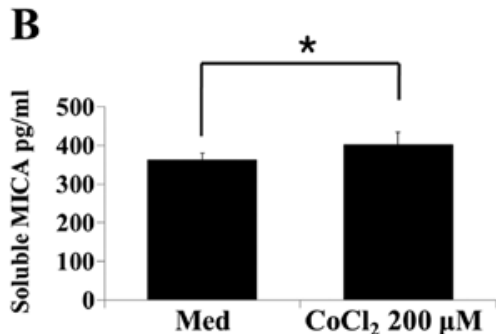

D

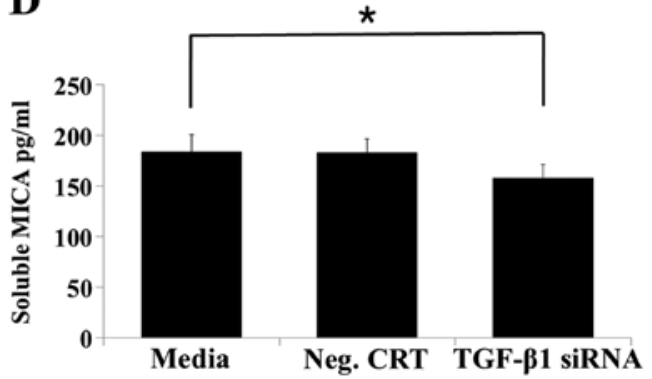

Figure 5. Production of soluble $\mathrm{MICA}$ from human renal proximal tubular epithelial cells following treatment with the hypoxia-mimetic chemical $\left(\mathrm{CoCl}_{2}\right)$ and TGF- $\beta$ gene silencing. (A) HK- 2 cells were incubated with or without $200 \mu \mathrm{M} \mathrm{CoCl}_{2}$ for $48 \mathrm{~h}$, and subsequently the TGF- $\beta$ and MICA mRNA levels were analyzed by RT-PCR. (B) HK-2 cells were treated with or without $\mathrm{CoCl}_{2}(200 \mu \mathrm{M})$ for $48 \mathrm{~h}$. MICA secretion into the culture supernatants was analyzed by ELISA. (C) HK- 2 cells were transfected with a control siRNA or TGF- $\beta$ siRNA, and RT-PCR was performed to determine the levels of TGF- $\beta$ and MICA. (D) HK-2 cells were transfected with a control siRNA or TGF- $\beta$ siRNA and maintained for 48 h. Following incubation, culture supernatants were harvested, and the level of soluble MICA in the culture supernatants was analyzed by ELISA. Results are representative of three independent experiments. ${ }^{*}<0.05$ vs. control. MICA, major histocompatibility complex class I-related chain molecules A; $\mathrm{CoCl}_{2}$, cobalt chloride; TGF, transforming growth factor; RT-PCR, reverse transcription polymerase chain reaction; ELISA, enzyme-linked immunosorbent assay; siRNA, small interfering RNA.

anti-Fas or isotype control antibodies did not (Fig. 4B). These results suggest that TGF- $\beta$ enhances NK cell cytotoxicity via an NKG2D-mediated pathway.

As MICA is expressed on the cell surface and released by metalloproteases $(38,39)$, the effect of metalloprotease treatment on TGF- $\beta$-induced MICA surface expression was evaluated. ELISA analysis of culture supernatants revealed that the shedding of MICA from TGF- $\beta$-treated HK- 2 cells was significantly enhanced at $72 \mathrm{~h}$ compared to that of the cells treated with media alone (Fig. 4C).

To determine the function of soluble MICA released from TGF- $\beta$-treated HK-2 cells, whether co-culture of TGF- $\beta$-treated HK-2 cells and NK cells affected the cytotoxicity of NK cells was evaluated using a Transwell system. Co-cultivation of NK cells with TGF- $\beta$-treated HK- 2 cells resulted in significantly lower NK cell cytotoxicity compared with the TGF- $\beta$-untreated HK-2 cells (Fig. 4D). Additionally, NK cells co-cultured with TGF- $\beta$-treated HK- 2 cells, in contrast to the NK cells that were not co-cultured, showed reduced NK activity against TGF- $\beta$-untreated HK-2 target cells. These results suggest that soluble MICA released from TGF- $\beta$-treated HK- 2 cells can reduce NK cell cytotoxicity via downregulation of NKG2D expression.

Hypoxia-induced TGF- $\beta$ promotes secretion of soluble MICA from renal proximal TECs. To confirm that the secreted soluble MICA observed in the HK-2 cell culture supernatant was due to hypoxia-induced TGF- $\beta$ expression, a hypoxia-mimetic chemical $\left[200 \mu \mathrm{M}\right.$ cobalt chloride $\left.\left(\mathrm{CoCl}_{2}\right)\right]$ was added to the HK-2 cells (40). $\mathrm{CoCl}_{2}$ enhanced expression of TGF- $\beta$ and MICA, as well as the release of soluble MICA from
HK-2 cells (Fig. 5A and B). To elucidate the direct effect of TGF- $\beta$ on soluble MICA secretion, TGF- $\beta$ gene silencing was performed using siRNA transfection (Fig. 5C) and confirmed by RT-PCR. Soluble MICA secretion corresponded with the level of TGF- $\beta$ silencing and was lower in TGF- $\beta$ siRNA transfected cells compared to the control and negative control siRNA-transfected cells (Fig. 5D). This result indicated that hypoxia-enhanced TGF- $\beta$ expression causes an increase in soluble MICA secretion, which in turn directly regulates IRI.

\section{Discussion}

TGF- $\beta$ is a multifunctional cytokine that induces cell apoptosis, differentiation and proliferation (26). Previous studies have reported that TGF- $\beta$ expression is induced throughout the kidney following renal IRI (27), and TGF- $\beta$ and its receptor system have been predominantly detected in local regenerative tubule cells $(27,28)$. Furthermore, several growth factors, including epidermal growth factor, platelet-derived growth factor, and basic fibroblast growth factor, have been shown to induce TGF- $\beta$ production in various renal cell cultures $(41,42)$. In addition, auto-induction of TGF- $\beta$ has also been observed in renal TECs (43); however, the function of TGF- $\beta$ in renal injury is not well understood. Recently, expression of TGF- $\beta$ detected in TECs has been shown to increase TEC survival following ischemic injury via upregulation of anti-apoptotic gene expression (30). Lee et al (44) reported that TGF- $\beta$ is involved in the mechanism of protection against $\mathrm{H}_{2} \mathrm{O}_{2}$-induced HK-2 cells death and the reduction of cellular events associated with renal IRI. These studies suggest that TGF- $\beta$ has a critical role in renal protection during renal IRI. 
Recently, NK cells have been linked to kidney IRI through direct targeting and lysis of TECs $(16,17)$, and induction of NKG2D ligand Rae-1 expression on TECs has been observed during kidney IRI (18). The present study shows that TGF- $\beta$ provides protection against NK cell killing, suggesting that TGF- $\beta$ modulates NK cell function in kidney IRI. It is well known that TGF- $\beta$ downregulates surface expression of NKG2D in NK cells $(24,25)$. In addition, Park et al (34) recently reported that TGF- $\beta$ decreased DAP10, which in turn led to the downregulation of NKG2D expression. Additionally, numerous cancer cells possess the ability to internalize the expression of NKG2D through binding of soluble MICA, representing another mechanism by which cancer cells can evade NK cell cytotoxicity $(38,39)$. Although TGF- $\beta$-induced membrane-bound MICA expression on HK-2 cells is critical for their susceptibility to NK cells, shedding soluble MICA can lead to internalization of NKG2D on NK cells allowing HK-2 cell survival (Figs. 4 and 5). Additionally, TGF- $\beta$ knockdown decreased production of soluble MICA in HK-2 cells (Fig. 5). On the basis of the direct effects of TGF- $\beta$-induced soluble MICA on NKG2D expression, we speculate that TGF- $\beta$ has an important role in the survival of renal proximal TECs through regulation of NKG2D expression in NK cells.

While induction of NKG2D ligands expression on renal proximal TECs during renal IRI has been reported previously in mouse models $(17,45)$, this event has not yet been observed in a human model of IRI. In general, kidney recipient patients are treated with immunosuppressive agents, including cyclosporine A, prior to kidney transplantation. As the immunological responses of kidney recipients are suppressed by the immunosuppressive agents, the levels of TGF- $\beta$ and soluble MICA that are detected within the serum of kidney transplantation patients cannot be considered accurate. However, Nakamura et al (40) have reported that the hypoxia-mimetic chemical $\mathrm{CoCl}_{2}$ can directly induce the production of angiogenic factors in cultured renal proximal tubular epithelial cells and that these factors have a critical role in the suppression of progressive renal damage. The present study showed that a hypoxia-mimetic chemical directly induced TGF- $\beta$ and MICA expression and enhanced production of soluble MICA in HK-2 cells (Fig. 5A and B). These results suggest that the induction of TGF- $\beta$ and MICA expression by hypoxia is a critical link between hypoxia and immune regulation during IRI.

Expression of NKG2D ligands can generally be induced in response to stress-associated signaling, including viral infection and malignant transformation $(46,47)$. Notably, several studies have reported that tissue ischemia can lead to upregulation of NKG2D ligands (17,18); however, the signaling pathways responsible for this upregulation following renal IRI are only partially understood. Numerous studies have shown that NKG2D ligand expression can be regulated at the transcriptional and posttranscriptional levels (48). Previously, several studies have shown that the ATM/ATR pathway is involved in the upregulation of NKG2D ligands following the DNA damage response $(35,36)$. Zhang et al (49) reported that ATM and p53 activation by TGF- $\beta$ occurred during apoptosis of epithelial cells. In addition, TGF- $\beta$-regulated ATM activity in response to genotoxic stress has also been reported (37). In the present study, expression of MICA was upregulated in renal TECs following treatment with TGF- $\beta$, while caffeine, an ATM/ATR kinase inhibitor, strongly

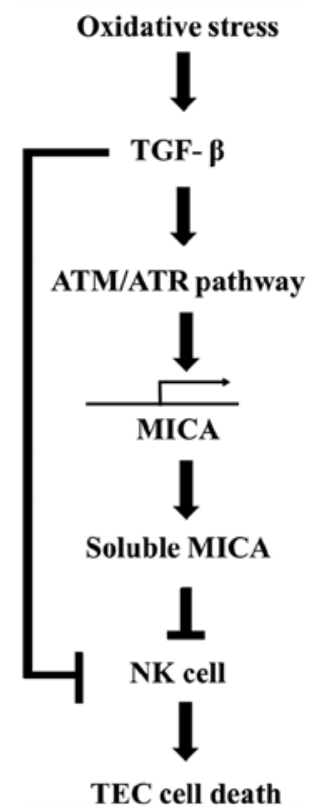

Figure 6. Schematic model depicting that TGF- $\beta$ regulate NK cells via production of soluble MICA in human renal proximal tubular epithelial cells based on the results of the present study. TGF, transforming growth factor; ATM, ataxia telangiectasia mutated; ATR, ATM- and Rad3-related; MICA, major histocompatibility complex class I-related chain molecules A; TEC, tubular epithelial cell.

inhibited this expression (Fig. 3). These findings suggest that TGF- $\beta$ regulates expression of MICA through activation of the ATM/ATR pathway in HK-2 cells.

Although membrane-bound MICA is critical for elimination of MICA-expressing cells through activation of NKG2D on $\mathrm{NK}, \mathrm{CD} 8^{+} \mathrm{T}$ and $\gamma \delta \mathrm{T}$ cells, numerous types of cell can affect survival through shedding of surface MICA by various proteases $(38,39)$. TGF- $\beta$ enhances the production of extracellular matrix proteins, and certain studies have reported that TGF- $\beta$ can also promote the expression of various proteases in HK-2 cells $(50,51)$. In the present study, soluble MICA secretion was strongly increased in the culture supernatant of TGF- $\beta$-treated HK- 2 cells (Fig. 4C). Therefore, it is possible that TGF- $\beta$ triggers the release of soluble MICA from HK-2 cells, although this should be investigated further in future studies.

In conclusion, TGF- $\beta$ induces MICA expression and its release from human renal proximal TECs, thereby regulating NKG2D expression on NK cells. The stability of MICA cell surface expression and the amount of soluble MICA released appear to affect the susceptibility of the target cells to NK cytotoxicity during renal IRI (Fig. 6). Modulation of surface and soluble MICA expression may provide a novel therapeutic target to improve graft survival following transplantation during renal IRI.

\section{Acknowledgements}

The present study was supported by a grant from the National R\&D Program for Cancer Control, Ministry for Health, Welfare and Family Affairs, Republic of Korea (grant no. 0920060) and by the Basic Science Research Program 
through the National Research Foundation of Korea (NRF) funded by the Ministry of Education, Science and Technology (grant no. 2011-0010291).

\section{References}

1. Shoskes DA and Halloran PF: Delayed graft function in renal transplantation: Etiology, management and long-term significance. J Urol 155: 1831-1840, 1996.

2. Thadhani R, Pascual M and Bonventre JV: Acute renal failure. N Engl J Med 334: 1448-1460, 1996.

3. Nigwekar SU, Kandula P, Hix JK and Thakar CV: Off-pump coronary artery bypass surgery and acute kidney injury: A meta-analysis of randomized and observational studies. Am J Kidney Dis 54: 413-423, 2009.

4. Perico N, Cattaneo D, Sayegh MH and Remuzzi G: Delayed graft function in kidney transplantation. Lancet 364: 1814-1827, 2004.

5. Bonventre JV and Weinberg JM: Recent advances in the pathophysiology of ischemic acute renal failure. J Am Soc Nephrol 14: 2199-2210, 2003

6. Boros P and Bromberg JS: New cellular and molecular immune pathways in ischemia/reperfusion injury. Am J Transplant 6 : 652-658, 2006

7. Molitoris BA and Sutton TA: Endothelial injury and dysfunction: Role in the extension phase of acute renal failure. Kidney Int 66 : 496-499, 2004

8. Kaminski KA, Bonda TA, Korecki J and Musial WJ: Oxidative stress and neutrophil activation--the two keystones of ischemia/reperfusion injury. Int J Cardiol 86: 41-59, 2002

9. Du C, Jiang J, Guan Q, Yin Z, Masterson M, Parbtani A, Zhong R and Jevnikar AM: Renal tubular epithelial cell self-injury through Fas/Fas ligand interaction promotes renal allograft injury. Am J Transplant 4: 1583-1594, 2004.

10. Du C, Wang S, Diao H, Guan Q, Zhong R and Jevnikar AM Increasing resistance of tubular epithelial cells to apoptosis by shRNA therapy ameliorates renal ischemia-reperfusion injury. Am J Transplant 6: 2256-2267, 2006.

11. Ascon DB, Lopez-Briones S, Liu M, Ascon M, Savransky V, Colvin RB, Soloski MJ and Rabb H: Phenotypic and functional characterization of kidney-infiltrating lymphocytes in renal ischemia reperfusion injury. J Immunol 177: 3380-3387, 2006.

12. Burne-Taney MJ, Ascon DB, Daniels F, Racusen L, Baldwin W and $\mathrm{Rabb} \mathrm{H}$ : $\mathrm{B}$ cell deficiency confers protection from renal ischemia reperfusion injury. J Immunol 171: 3210-3215, 2003.

13. Dong X, Swaminathan S, Bachman LA, Croatt AJ, Nath KA and Griffin MD: Resident dendritic cells are the predominant TNF-secreting cell in early renal ischemia-reperfusion injury. Kidney Int 71: 619-628, 2007.

14. Burne-Taney MJ, Yokota-Ikeda $\mathrm{N}$ and Rabb H: Effects of combined T- and B-cell deficiency on murine ischemia reperfusion injury. Am J Transplant 5: 1186-1193, 2005.

15. Park P, Haas M, Cunningham PN, Bao L, Alexander JJ and Quigg RJ: Injury in renal ischemia-reperfusion is independent from immunoglobulins and T lymphocytes. Am J Physiol Renal Physiol 282: F352-F357, 2002

16. Zhang ZX, Shek K, Wang S, Huang X, Lau A, Yin Z, Sun H, Liu W, Garcia B, Rittling S, et al: Osteopontin expressed in tubular epithelial cells regulates NK cell-mediated kidney ischemia reperfusion injury. J Immunol 185: 967-973, 2010.

17. Zhang ZX, Wang S, Huang X, Min WP, Sun H, Liu W, Garcia B and Jevnikar AM: NK cells induce apoptosis in tubular epithelial cells and contribute to renal ischemia-reperfusion injury. J Immunol 181: 7489-7498, 2008.

18. Feng L, Cheng F, Ye Z, Li S, He Y, Yao X, Tang Q and Li Y: The effect of renal ischemia-reperfusion injury on expression of RAE-1 and H60 in mice kidney. Transplant Proc 38: 2195-2198, 2006.

19. Moretta A, Marcenaro E, Parolini S, Ferlazzo G and Moretta L: NK cells at the interface between innate and adaptive immunity. Cell Death Differ 15: 226-233, 2008.

20. Schmitt C, Ghazi B and Bensussan A: NK cells and surveillance in humans. Reprod Biomed Online 16: 192-201, 2008.

21. Raulet DH: Interplay of natural killer cells and their receptors with the adaptive immune response. Nat Immunol 5: 996-1002, 2004.

22. Raulet DH: Roles of the NKG2D immunoreceptor and its ligands. Nat Rev Immunol 3: 781-790, 2003.
23. Welte SA, Sinzger C, Lutz SZ, Singh-Jasuja H, Sampaio KL, Eknigk U, Rammensee HG and Steinle A: Selective intracellular retention of virally induced NKG2D ligands by the human cytomegalovirus UL16 glycoprotein. Eur J Immunol 33: 194-203, 2003.

24. Crane CA, Han SJ, Barry JJ, Ahn BJ, Lanier LL and Parsa AT: TGF-beta downregulates the activating receptor NKG2D on NK cells and $\mathrm{CD}^{+} \mathrm{T}$ cells in glioma patients. Neuro Oncol 12: 7-13, 2010.

25. Lee JC, Lee KM, Kim DW and Heo DS: Elevated TGF-betal secretion and down-modulation of NKG2D underlies impaired NK cytotoxicity in cancer patients. J Immunol 172: 7335-7340, 2004.

26. Siegel PM and Massagué J: Cytostatic and apoptotic actions of TGF-beta in homeostasis and cancer. Nat Rev Cancer 3: 807-821, 2003.

27. Basile DP, Rovak JM, Martin DR and Hammerman MR: Increased transforming growth factor-beta 1 expression in regenerating rat renal tubules following ischemic injury. Am J Physiol 270: F500-F509, 1996.

28. Gobé G, Willgoss D, Hogg N, Schoch E and Endre Z: Cell survival or death in renal tubular epithelium after ischemia-reperfusion injury. Kidney Int 56: 1299-1304, 1999.

29. Docherty NG, Pérez-Barriocanal F, Balboa NE and López-Novoa JM: Transforming growth factor-beta1 (TGF-beta1): A potential recovery signal in the post-ischemic kidney. Ren Fail 24: 391-406, 2002.

30. Guan Q, Nguan CY and Du C: Expression of transforming growth factor-betal limits renal ischemia-reperfusion injury. Transplantation 89: 1320-1327, 2010.

31. Luo L, Lu J, Wei L, Long D, Guo JY, Shan J, Li FS, Lu PY, Li PY and Feng L: The role of HIF-1 in up-regulating MICA expression on human renal proximal tubular epithelial cells during hypoxia/reoxygenation. BMC Cell Biol 11: 91, 2010.

32. Huenecke S, Zimmermann SY, Kloess S, Esser R, Brinkmann A, Tramsen L, Koenig M, Erben S, Seidl C, Tonn T, et al: IL-2-driven regulation of NK cell receptors with regard to the distribution of $\mathrm{CD}^{-16^{+}}$and $\mathrm{CD} 16^{-}$subpopulations and in vivo influence after haploidentical NK cell infusion. J Immunother 33: 200-210, 2010.

33. Lichtenfels R, Biddison WE, Schulz H, Vogt AB and Martin R CARE-LASS (calcein-release-assay), an improved fluorescence-based test system to measure cytotoxic T lymphocyte activity. J Immunol Methods 172: 227-239.

34. Park YP, Choi SC, Kiesler P, Gil-Krzewska A, Borrego F, Weck J, Krzewski $\mathrm{K}$ and Coligan JE: Complex regulation of human NKG2D-DAP10 cell surface expression: Opposing roles of the $\gamma c$ cytokines and TGF- $\beta 1$. Blood 118: 3019-3027, 2011.

35. Cerboni C, Zingoni A, Cippitelli M, Piccoli M, Frati L and Santoni A: Antigen-activated human T lymphocytes express cell-surface NKG2D ligands via an ATM/ATR-dependent mechanism and become susceptible to autologous $\mathrm{NK}^{-}$cell lysis. Blood 110: 606-615, 2007

36. Lu X, Ohata K, Kondo Y, Espinoza JL, Qi Z and Nakao S: Hydroxyurea upregulates NKG2D ligand expression in myeloid leukemia cells synergistically with valproic acid and potentially enhances susceptibility of leukemic cells to natural killer cell-mediated cytolysis. Cancer Sci 101: 609-615, 2010.

37. Kirshner J, Jobling MF, Pajares MJ, Ravani SA, Glick AB, Lavin MJ, Koslov S, Shiloh Y and Barcellos-Hoff MH: Inhibition of transforming growth factor-beta1 signaling attenuates ataxia telangiectasia mutated activity in response to genotoxic stress. Cancer Res 66: 10861-10869, 2006.

38. Salih HR, Goehlsdorf D and Steinle A: Release of MICB molecules by tumor cells: Mechanism and soluble MICB in sera of cancer patients. Hum Immunol 67: 188-195, 2006

39. Salih HR, Rammensee HG and Steinle A: Cutting edge: Down-regulation of MICA on human tumors by proteolytic shedding. J Immunol 169: 4098-4102, 2002.

40. Nakamura M, Yamabe H, Osawa H, Nakamura N, Shimada M, Kumasaka R, Murakami R, Fujita T, Osanai T and Okumura K Hypoxic conditions stimulate the production of angiogenin and vascular endothelial growth factor by human renal proximal tubular epithelial cells in culture. Nephrol Dial Transplant 21: 1489-1495, 2006

41. Di Paolo S, Gesualdo L, Ranieri E, Grandaliano G and Schena FP: High glucose concentration induces the overexpression of transforming growth factor-beta through the activation of a platelet-derived growth factor loop in human mesangial cells. Am J Pathol 149: 2095-2106, 1996. 
42. Yamabe H, Osawa H, Kaizuka M, Tsunoda S, Shirato K, Tateyama F and Okumura K: Platelet-derived growth factor, basic fibroblast growth factor, and interferon gamma increase type IV collagen production in human fetal mesangial cells via a transforming growth factor-beta-dependent mechanism. Nephrol Dial Transplant 15: 872-876, 2000.

43. Dockrell ME, Phanish MK and Hendry BM: Tgf-beta auto-induction and connective tissue growth factor expression in human renal tubule epithelial cells requires $\mathrm{N}$-ras. Nephron, Exp Nephrol 112: e71-e79, 2009.

44. Lee HT, Kim M, Kim J, Kim N and Emala CW: TGF-beta1 release by volatile anesthetics mediates protection against renal proximal tubule cell necrosis. Am J Nephrol 27: 416-424, 2007.

45. Chen GE, Wu H, Ma J, Chadban SJ and Sharland A: Toll-like receptor 4 engagement contributes to expression of NKG2D ligands by renal tubular epithelial cells. Nephrol Dial Transplant 26: 3873-3881, 2011.

46. Diefenbach A, Jensen ER, Jamieson AM and Raulet DH: Rae1 and H60 ligands of the NKG2D receptor stimulate tumour immunity. Nature 413: 165-171, 2001.
47. Groh V, Bahram S, Bauer S, Herman A, Beauchamp M and Spies T: Cell stress-regulated human major histocompatibility complex class I gene expressed in gastrointestinal epithelium. Proc Natl Acad Sci USA 93: 12445-12450, 1996.

48. López-Soto A, Folgueras AR, Seto E and Gonzalez S: HDAC3 represses the expression of NKG2D ligands ULBPs in epithelial tumour cells: Potential implications for the immunosurveillance of cancer. Oncogene 28: 2370-2382, 2009.

49. Zhang S, Ekman M, Thakur N, Bu S, Davoodpour P, Grimsby S, Tagami S, Heldin CH and Landström M: TGFbeta1-induced activation of ATM and p53 mediates apoptosis in a Smad7-dependent manner. Cell Cycle 5: 2787-2795, 2006.

50. Deng B,Yang X,Liu J,HeF,ZhuZandZhang C:Focal adhesionkinase mediates TGF-beta1-induced renal tubular epithelial-to-mesenchymal transition in vitro. Mol Cell Biochem 340: 21-29, 2010.

51. Wang QL, Tao YY, Yuan JL, Shen L and Liu CH: Salvianolic acid B prevents epithelial-to-mesenchymal transition through the TGF-betal signal transduction pathway in vivo and in vitro. BMC Cell Biol 11: 31, 2010. 